\title{
Study on parching procedure - acid leaching of Yunnan phosphogypsum decolorization mechanism based on Origin software
}

\author{
Ziyu Liu", a, Zhiman Zhao, b, Chenxi Du, c, Shuai $\mathrm{Li}^{1, \mathrm{~d}}$ \\ ${ }^{1}$ Faculty of Architecture and Engineering, Kunming University of Science and Technology, Kunming, \\ 650500, Yunnan, China

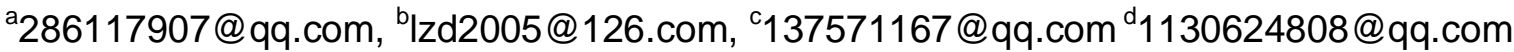

\begin{abstract}
Key words:Phosphogypsum;Parching procedure-acid leaching;Decolorization;Origin;Curve fitting Abstract. By the method of parching procedure and acid leaching to improve the phosphogypsum whiteness, and take advantage of the curve fitting technique of the Origin software to fitting the experimental data. Than, get the related curves and equation expression of the parching procedure and phosphogypsum whiteness during the heating process. Thereby providing a solution for the determination of the parching temperature. The results show that use curve fitting equation obtained calculate the required whiteness desired parching temperature. And calculate the range of the equation expression, that parching temperature control at $0{ }^{\circ} \mathrm{C}-800{ }^{\circ} \mathrm{C}$, whiteness range at $68.6 \%$ $81.72 \%$. Further increase parching time and change leaching conditions, the phosphogypsum whiteness can be increased.
\end{abstract}

\section{Introduction}

Phosphogypsum was discharged from wet phosphoric acid production of solid waste, was the largest number of industrial by-product gypsum, phosphogypsum annual emissions of phosphorus chemical industry more than $70 \%$ of total industrial by-product gypsum, to about 50 million $\mathrm{t}$, and maintain a $15 \%$ annual growth rate ${ }^{[1-4]}$ 。 And the accumulation of phosphogypsum in place, large area, high slag heap and administrative costs, caused enormous environmental pollution ${ }^{[5-8]}$ 。

The main component of phosphogypsum as $\mathrm{CaSO}_{4} \cdot 2 \mathrm{H}_{2} \mathrm{O}$, it contains organic matter and a small amount of $\mathrm{SiO}_{2} 、 \mathrm{Al}_{2} \mathrm{O}_{3} 、 \mathrm{Fe}_{2} \mathrm{O}_{3} 、 \mathrm{CaO} 、 \mathrm{MgO}$ and other impurities, usually pale yellow, light gray or dark gray, low brightness, largely limited it is used in paint and decorative products. With the advance of resource requirements of sustainable development, to reduce a lot of the development of natural resources, combined cycle applications of phosphogypsum was particularly important. With the development of the times, people demanded more and more houses decorated high, especially for the development of interior painting and decorative products in the construction of gypsum plaster products in whiteness has certain requirements, so whitening phosphogypsum Research increasingly being taken seriously ${ }^{[9-10]}$.

By Origin software phosphogypsum parching temperature curve Gauss curve fitting temperature curve equation expressions, which provides a new way to shorten experiment time and saving experimental materials, but also for industrial parching phosphogypsum powder production building control its whiteness provides advanced scientific methods.

\section{Experimental}

\section{Main materials and reagents.}

Phosphogypsum of Yunnan Yuntianhua limited liability company. Original whiteness $32.8 \%$, analysis of the main chemical components shown in Table 1.

Table 1 Phosphogypsum component

\begin{tabular}{lcccccccccc}
\hline component & $\mathrm{SO}_{3}$ & $\mathrm{P}_{2} \mathrm{O}_{5}$ & $\mathrm{H}_{2} \mathrm{O}$ & $\mathrm{Al}_{2} \mathrm{O}_{3}$ & $\mathrm{SiO}_{2}$ & $\mathrm{CaO}$ & $\mathrm{F}$ & $\mathrm{Cl}$ & $\mathrm{Fe}_{2} \mathrm{O}_{3}$ & $\mathrm{MgO}$ \\
\hline Percentage /\% & 44.73 & 0.71 & 6.32 & 0.75 & 14.34 & 24.92 & 0.17 & 0.016 & 0.41 & 0.031 \\
\hline Reagents: sulfuric acid
\end{tabular}

Reagents: sulfuric acid, analytical grade,Shanghai Chemical Reagent Company. 


\section{Main instrument.}

Experimental main instruments are shown in Table 2.

Table 2 The main instrument

\begin{tabular}{ccc}
\hline Equipment & Type & Manufacturer \\
\hline Universal oven & $220 \mathrm{~V}, 1 \mathrm{KW}$ & Beijing Zhongxing Weiye Instrument Co., Ltd. \\
White tester & WSB-2 & Hangzhou Qiwei Instrument Co., Ltd. \\
Hot blast oven & $101-4$ & Shanghai Guangdi Instrument Co., Ltd. \\
Rotary vane vacuum pump & XZ-0.5B & Zhengzhou Zhongtian Experimental Equipment \\
\hline
\end{tabular}

\section{Experimental Procedure.}

(1)Phosphogypsum weighed a certain amount of milled in a crucible, with universal furnace at a certain temperature parching some time,cooled to room temperature and standby.

(2) After parching phosphogypsum with sulfuric acid solution after a certain amount of content and put in the three-necked flask, At a certain temperature for stirring time, after a sufficient reaction time are determined, filtration, washing, drying, grinding, the resulting sample whiteness (W) Test. As shown in Table 3.

Table 3 the different temperature corresponded whiteness

\begin{tabular}{cccccccc}
\hline Temp $/{ }^{\circ} \mathrm{C}$ & 100 & 150 & 200 & 250 & 300 & 350 & 400 \\
\hline \multirow{3}{*}{ Whiteness $/ \%$} & 66.6 & 68.4 & 72.4 & 75.3 & 78.5 & 79.9 & 81.2 \\
& 66.2 & 68.5 & 71.6 & 76.2 & 78.6 & 80.5 & 81.3 \\
$\begin{array}{c}\text { The average } \\
\text { of whiteness }\end{array}$ & 66.4 & 68.6 & 71.6 & 76.0 & 78.6 & 80.7 & 81.2 \\
\hline
\end{tabular}

\section{Sampling criterion.}

The sample obtained in strict accordance with "GB / T 5950-2008 building materials and non-metallic mineral products whiteness measurement method" sample to measure its whiteness.

\section{Origin data fitting and analysis}

\section{Data fitting.}

Origin widely used in scientific research in the field of data analysis and mapping, it not only has a strong linear regression and curve fitting functions, as well as powerful graphics capabilities, using the software can reduce the randomness of data processing, reduce errors, fast and convenient . But also more reasonable results ${ }^{[1]}$.Figure 1 shows, parching temperature and brightness are nonlinear characteristics.

Origin of experimental results utilizing nonlinear fitting custom function capabilities of whiteness different parching temperatures nonlinear curve fitting fitting function, while reducing data processing error, it can easily and quickly obtain the desired. This was to solve a lot of trial number of great help ${ }^{[12]}$.

The data in Table 3 through Origin converted into two-dimensional graphics processing Line + Symbol is shown in Figure 1. 


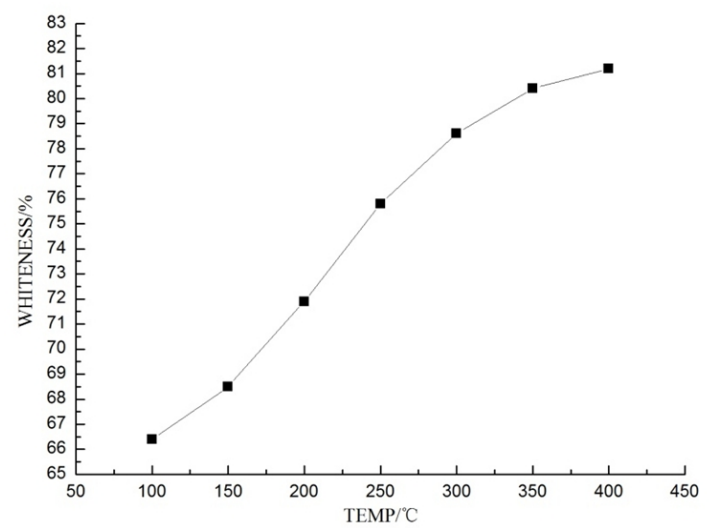

Fig.1 Relationship curve of parching procedure temperature and whiteness

Exhibit nonlinear characteristics curve during heating according to adopt non-linear fitting method Guass fitting result by Origin software:

$$
\begin{aligned}
\mathrm{y}= & -\frac{4657.92}{240.85 \sqrt{\pi / 2}} \mathrm{e}^{-2 \frac{(\mathrm{x}-80.2)^{2}}{240.85^{2}}}+81.72 \\
& \mathrm{x} \text { - temperature; } \\
& \mathrm{y} \text { - whiteness; }
\end{aligned}
$$

Origin of the effectivity analysis equation, goodness of fit (Adj.R-square) as follows: 0.99979. Residual sum of squares (Ruduced Chi-sqr) as follows: 0.00718. Explained fit well. The resulting fitted curve comparison with the experimental data shown in Figure 2.

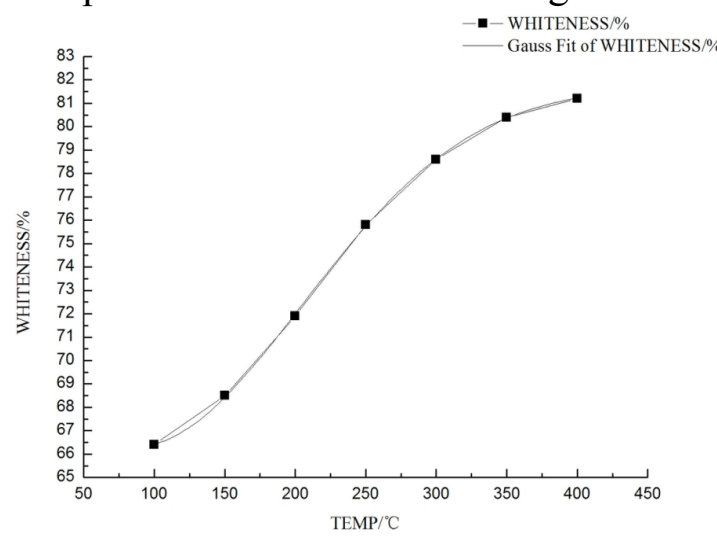

Fig. 2 The experimental curve and the fittng curve y

\section{Analysis.}

From Figure 2, curve fitting functions and experimental data in good condition. For fitting function (1) derivative calculations, was a function of the minimum value of $x=0.33, y=68.6$.

Expressions Gaussian curve is:

$$
y=\frac{1}{\sqrt{2 \pi \sigma}} \exp \left[\frac{-(x-\mu)^{2}}{2 \sigma^{2}}\right]
$$

$\mu_{\text {was mean value, }} \sigma$ was standard deviation. Through nonlinear fitting of experimental data with the origin of Gaussian (Gauss) got the $\mu=80.2, \sigma=240.85$. To determine the coefficient obtained equation expression(1). Gauss function, also known as the Gaussian distribution, are normal distribution, it can be seen from Figure 2, the curve rises gradually, to get fit function (1) calculated to get the maximum whiteness of $81.72 \%$, and on the basis of experimental data fitting Gaussian distribution curve obtained. When the temperature exceeds $800{ }^{\circ} \mathrm{C}$, will make the whiteness of phosphogypsum reduced, or even fire phenomenon. Want to get the actual production of certain 
white phosphogypsum building plaster will need to do a lot of experimental, which will undoubtedly increase the number of experiments.

Taking the logarithm of function (1) give functional relationship (3).

$$
\begin{aligned}
\mathrm{y}= & \sqrt{-\frac{1}{2}(24085)^{2} \operatorname{In}\left[-\frac{24085 \sqrt{\pi / 2}(x-81.72)}{465792}\right]}+80.2 \\
& \mathrm{x} \text { - whiteness; } 65<\mathrm{x}<81.72 ;
\end{aligned}
$$

In production, reduce product cost was an important part of the production process to determine solutions. Therefore, in the production of the resulting fitting function can be used (3) according to the desired brightness value can be determined phosphogypsum heating temperature, such as to give certain whiteness phosphogypsum $\mathrm{x}$, using the function (3) can be obtained the required parching temperature y. To verify the function (3), the election of the desired whiteness was $70 \%, 75 \%, 78 \%$, $80 \%, 81 \%$, and calculate the corresponding parching temperature for verification experiments in this parching temperature. Experimental data obtained with function (3) comparing the calculated values,

\begin{tabular}{|c|c|c|c|c|c|c|}
\hline \multirow{2}{*}{$\begin{array}{l}\text { Calculated } \\
\text { value }\end{array}$} & whiteness $/ \%$ & 70 & 75 & 78 & 80 & 81 \\
\hline & temperature $/{ }^{\circ} \mathrm{C}$ & 181.26 & 242.5 & 288.76 & 336.85 & 382.08 \\
\hline \multirow{5}{*}{$\begin{array}{l}\text { Experimental } \\
\text { value }\end{array}$} & & 70.7 & 76.1 & 77.9 & 80.1 & 80.8 \\
\hline & whiteness $/ \%$ & 70.3 & 76.2 & 77.3 & 79.8 & 80.7 \\
\hline & & 70.7 & 75.9 & 78.2 & 79.9 & 81.3 \\
\hline & $\begin{array}{c}\text { Whiteness average } \\
\text { value } / \%\end{array}$ & 70.68 & 75.93 & 77.8 & 79.9 & 80.9 \\
\hline & temperature $/{ }^{\circ} \mathrm{C}$ & 180 & 250 & 290 & 340 & 380 \\
\hline
\end{tabular}
specific experimental data shown in Table 4.

Table 4 The different parching temperature and computed temperature values of whiteness

As can be seen from the desired whiteness Table 4, according to the function (3) Find the corresponding parching temperature, temperature control can get the desired whiteness. When the temperature is $0.33{ }^{\circ} \mathrm{C}$ can be drawn from the fitting function (1), corresponding to the whiteness of $68.6 \%$, and can be seen from Table 1 and Table 3 , at $0{ }^{\circ} \mathrm{C} \sim 200{ }^{\circ} \mathrm{C}$ parching phosphogypsum after the top after parching during acid leaching experiments, whiteness will increase, but has remained in the $60 \%$ to $70 \%$ of the low brightness range, and in the process of acid leaching acid infusion of foreign matter was appeared in a relatively at $320{ }^{\circ} \mathrm{C} \sim 430{ }^{\circ} \mathrm{C}$ parching phosphogypsum in acid leaching process does not occur this phenomenon. This shows that acid leaching process to increase the whiteness of phosphogypsum is crucial, and whiteness in a relatively high temperature above $250{ }^{\circ} \mathrm{C}$ range parching phosphogypsum after pickling increases significantly, so as to increase phosphogypsum should try to improve the whiteness parching temperature, as shown in Table 3. Try to choose high temperature, let phosphogypsum in a good burn and remove impurities, provide a good foundation for the back of the whitening process.

On the most commonly used heating devices such as wok rotary kiln, circulating fluidized bed and in suspension dewatering equipment, industrial, experiment procedures can be combined to improve the whiteness of phosphogypsum, only stage for parching, the parching pan relatively low temperature, can be appropriate to increase the parching time for phosphogypsum impurities in full combustion; High temperature rotary kiln and circulating fluidized bed boilers, high-temperature heating can be appropriately selected within the allowable temperature range; For the current most efficient dewatering process in suspension, may be appropriate to raise the temperature, impurities in phosphogypsum full combustion; Experiments show that want to get high brightness can be used parching and pickling combination of methods to improve the phosphogypsumwhiteness. 


\section{Conclusion}

1.Use Origin software to curve fitting, the experiment data processing can reduce errors, and can guide the experiment, reduce the amount of experimentation. Origin curve fitting techniques to obtain the use of parching temperature and whiteness curve and the curve equation obtained expressions, calculated the use of this expression, namely parching temperature control between $0^{\circ} \mathrm{C}$ to $800^{\circ} \mathrm{C}$, get the whiteness of the range of $68.6 \%-81.72 \%$. Reduces the number of experiments could save a lot of experiments to bring the economic and environmental problems, and can be used to guide industrial parching temperature and corresponding control measures.

2.In phosphogypsum whitening discolored study found that parching stage phosphogypsum impurities in gypsum good combustion, can provide a good foundation for the post-whitening process. Try to choose parching temperature above $200{ }^{\circ} \mathrm{C}$.

3.In the parching stage if the temperature is not enough, increase the time to let phosphogypsum parching impurities in full combustion. Use other heating equipment can be appropriate to raise the temperature within the appropriate temperature range of phosphogypsum is heated, so that impurities of phosphogypsum in more complete combustion. To get a higher whiteness phosphogypsum, take control of other variable factors to increase the whiteness, such as increasing the parching time, select the appropriate acid leach solution and the mass fraction .

\section{Acknowledgements}

This work was financially supported by the National Natural Science Foundation (51264017).

※Corresponding author: Zhao zhiman, provide phone: 86-13529317921; Fax: 86-0871-63864560

\section{Reference}

[1]J.M.Wang: China Petroleum and Chemical Industry Analysis.Vol.9(2012),P.52(In Chinese)

[2]W.Wu, X.Tong. The hydrometallurgical.Vol.29,No.3(2012),P139(In Chinese)

[3]R.H.Yang. Phosphorus Fertilization and Compound Fertilizer. Vol.23,No.6(2008),P139(In Chinese)

[4]L.Z.Ma, P.Ning. Phosphorus Fertilization and Compound Fertilizer. Vol.22,No.1(2007),P54(In Chinese)

[5]T.M.Guo. Industrial by-product gypsum Applications.Beijing. China Building Materials Industry Press. 2010:57-73(In Chinese)

[6]Hanan Tayibi, Mohamed Choura, Felix A. Lopez, et al. Environmental impact and management of phosphagypsum[J]. Journal of Environmental Management: 2009, Vol. 90: 2377-2386

[7]Q.F.Wang. Brick and tile. Vol.5(2008),P.57(In Chinese)

[8]E.M. ElAfifi, M.A. Hilal. Characterization of phosphogypsum wastes associated with phosphoric acid and fertilizers production., Journal of Environmental Radioactivity[J].2009,100:407-412

[9]M.Shi. Hefei University of Technology. Vol.36,No.2(2013),P212(In Chinese)

[10] J.S.Zhang. Non-metallic Minerals. Vol.27,No.6(2004),P44(In Chinese)

[11] C.L.Xia. University Chemistry. Vol.18,No.2(2003),P44(In Chinese)

[12]M.L.Xiang. Mianyang Normal University Learned Journal. Vol.23,No.2(2004),P61(In Chinese) 